\title{
Critical Neonatal Congenital Heart Disease - a Rare Complication after Successful Surgical Correction
}

\author{
Carmen Corina Șuteu ${ }^{1}$, Iolanda Muntean², Cristina Blesneac ${ }^{1}$, Marian Pop $^{2}$, Rodica Togănel ${ }^{2}$ \\ ${ }^{1}$ Department of Pediatric Cardiology, Emergency Institute for Cardiovascular Diseases and Transplantation, Târgu Mureș, Romania \\ 2 "George Emil Palade" University of Medicine, Pharmacy, Science and Technology, Târgu Mureș, Romania
}

\begin{abstract}
Pulmonary arterial hypertension (PAH) is a rare but severe complication that should be investigated in patients diagnosed with transposition of the great arteries who have undergone neonatal arterial switch operation. Early diagnosis and aggressive combination therapy for PAH could help to improve survival in these patients. We report a favorable clinical response 6 months after the initiation of vasodilator therapy in a pediatric case with transposition of the great arteries, successfully repaired in the neonatal period, who developed pulmonary arterial hypertension at the age of 5 years.
\end{abstract}

Keywords: pulmonary arterial hypertension, transposition of the great arteries

\section{ARTICLE HISTORY}

Received: October 14, 2020

Accepted: December 2, 2020

\section{CORRESPONDENCE}

Iolanda Muntean

Str. Gheorghe Marinescu nr. 50

540136 Târgu Mureș, Romania

Tel: +40 265215551

E-mail: iolanda.muntean@gmail.com

\section{INTRODUCTION}

Pulmonary arterial hypertension (PAH) is a rare and progressive disease of the pulmonary vasculature characterized by elevated pulmonary artery pressure (PAP). ${ }^{1}$ The prognosis is poor due to progressive right heart failure. In children, pulmonary hypertension ( $\mathrm{PH}$ ) is most associated with underlying cardiac or lung disease. ${ }^{1}$ Only a small percentage of patients with congenital heart disease (CHD) develop clinically significant PH. PH can persist and increase in patients after successful corrective surgery of cardiac lesions.

Transposition of the great arteries (TGA) is one of the more common cyanotic CHDs with an estimated preva- lence of 2.3 to 4.7 per 10,000 live births. ${ }^{2}$ Arterial switch operation (ASO), originally performed in 1975 , has become the standard corrective procedure for patients with TGA with intact interventricular septum (IVS) and without any other cardiac defects. ${ }^{3}$ It is usually performed within the first few weeks of life before the left ventricle becomes deconditioned and therefore unable to function as the systemic ventricle. All patients who have undergone surgical repair of TGA require long-term cardiology follow-up. Most of these patients remain free from long-term cardiovascular complications. ${ }^{4}$ The most common complication of ASO is pulmonary artery stenosis, which may require reintervention. Pulmonary arterial hypertension in TGA with IVS is extremely rare, but this serious complica- 
tion can occur even in patients undergoing an ASO before the age of 1 month. ${ }^{5-8}$ Vasodilator specific therapy should first be provided in these cases. ${ }^{9}$

Here we report a favorable clinical response at 6 months after the initiation of vasodilator therapy in a pediatric case of PAH after ASO.

\section{CASE REPORT}

We report the case of a 5-year-old girl diagnosed with TGA with IVS at birth. During the neonatal period there was no evidence of persistent pulmonary hypertension of the newborn (PPHN). Also, the patient did not undergo cardiac catheterization for Rashkind balloon atrial septostomy. When she was 13 days old, an ASO was performed. The patient was followed-up every 6 months in the pediatric cardiology department, with no clinical and echocardiographic signs that might have led to suspicion of $\mathrm{PH}$. Her clinical condition gradually deteriorated one month before admission, with World Health Organization (WHO) functional class (FC) III at presentation. On examination, her blood pressure was 105/81 $\mathrm{mmHg}$, the pulse rate was regular at 126/minute, and oxygen saturation was $96 \%$. Cardiac auscultation revealed a loud pulmonic second heart sound and a soft murmur indicating tricuspid regurgitation. The physical examination revealed mild hepatomegaly. In the 6-minute walk test, she covered 288 $\mathrm{m}$, and her heart rate increased from 95 to $145 \mathrm{bpm}$. The electrocardiogram showed sinus tachycardia with right axis deviation, right atrial dilation, and right ventricular (RV) hypertrophy. Echocardiography revealed septal flattening with left ventricular eccentricity index of 1.5, moderately dilated and mildly impaired RV, with tricuspid annular plane systolic excursion (TAPSE) of $10.5 \mathrm{~mm}$, with an estimated mean PAP of $63 \mathrm{mmHg}$ (Figure 1). No other abnormalities were suggestive of $\mathrm{PH}$. Laboratory assessment excluded connective tissue diseases, renal and liver diseases. There was no evidence of sleep apnea. Contrastenhanced computed tomography confirmed the presence of TGA corrected with ASO, and no residual obstructive lesions nor parenchymal lung lesions were observed (Figure 2). Cardiac catheterization revealed a right atrial pressure of $7 \mathrm{mmHg}$, a mean PAP of $44 \mathrm{mmHg}$, and a pulmonary capillary wedge pressure (PCWP) of $9 \mathrm{mmHg}$. The pulmo-

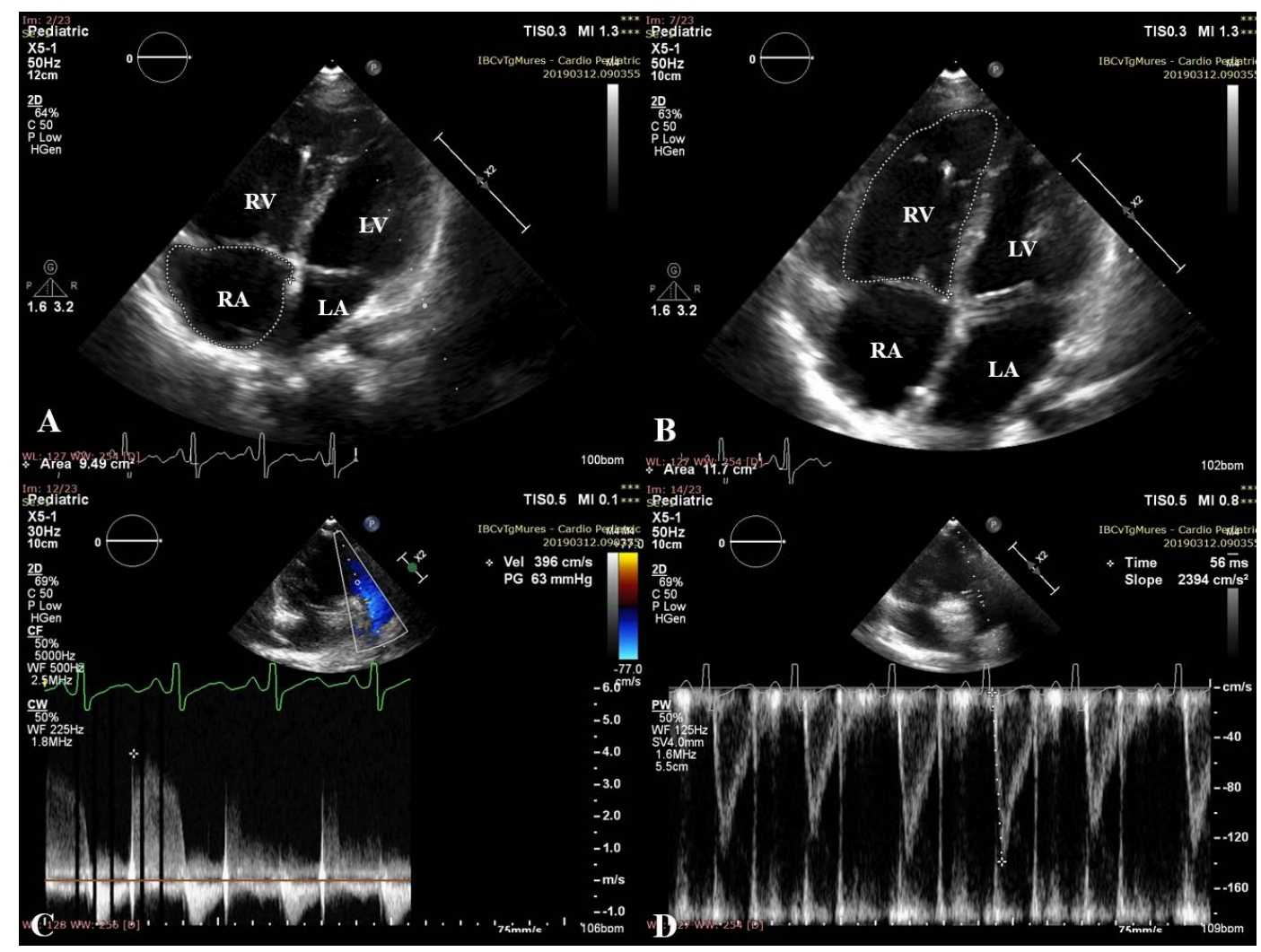

FIGURE 1. Echocardiographic views before initiation of vasodilator therapy demonstrating: $\mathbf{A}, \mathbf{B}$-dilation of the right cavities; A - right atrial area of $9.49 \mathrm{~cm}^{2}$; $\mathbf{B}$ - right ventricular area of $11.7 \mathrm{~cm}^{2}$; $\mathbf{C}$ - mean pulmonary arterial pressure of $63 \mathrm{mmHg}$; D - pulmonary artery acceleration time of $56 \mathrm{~ms}$. LA - left atrium; LV - left ventricle; RA - right atrium; RV - right ventricle 


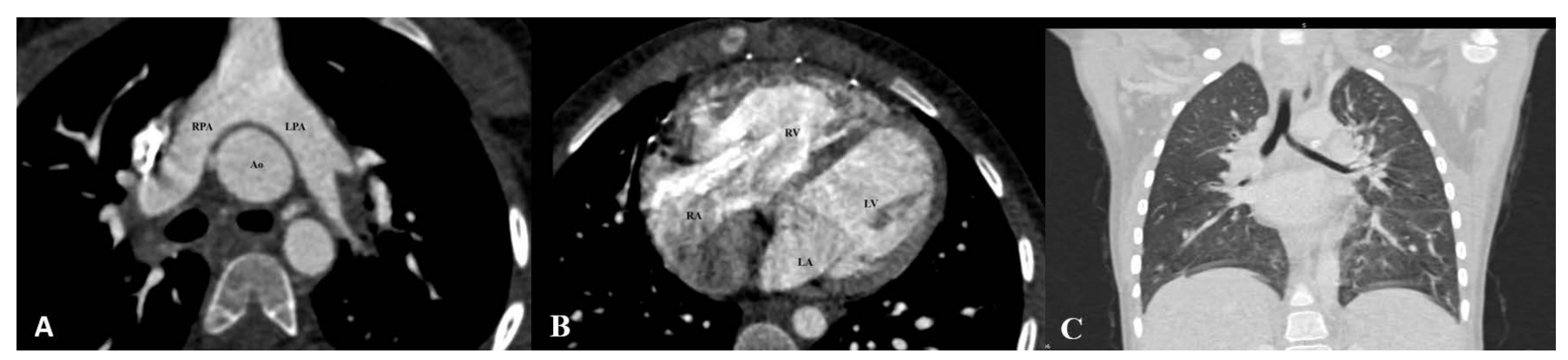

FIGURE 2. Computed tomography angiography scan showing: A - position of the pulmonary artery after switch arterial operation, without residual obstructive lesions; $\mathbf{B}$ - dilation of the right cavities; $\mathbf{C}$ - the absence of parenchymal lung lesions. Ao - aorta; LA - left atrium; LPA - left pulmonary artery; LV - left ventricle; RA - right atrium; RPA - right pulmonary artery; RV - right ventricle

nary vascular resistance (PVR) was 8.97 Wood units $/ \mathrm{m}^{2}$, with no acute vasodilatory response with inhaled NO. There was no significant demonstrable left-to-right or right-to-left shunt (Qp:Qs was 1).

In view of this diagnosis, associated therapy for $\mathrm{PAH}$ with bosentan $62.5 \mathrm{mg} / 12 \mathrm{~h}$ and sildenafil $10 \mathrm{mg} / 8 \mathrm{~h}$ was initiated.

After 6 months, clinical improvement was seen in terms of heart rate and the distance walked in 6 minutes; she covered $468 \mathrm{~m}$, and her heart rate increased from 76 to $108 \mathrm{bpm}$. Echocardiography revealed improvement of echocardiographic parameters that reflect the RV function and the hemodynamics of the right heart (Figure 3). At the time of this writing, the girl remains stable at 24 months (WHO FC II).

The publication of this case was approved by the institutional review board in the presence of a signed informed consent obtained from the mother.

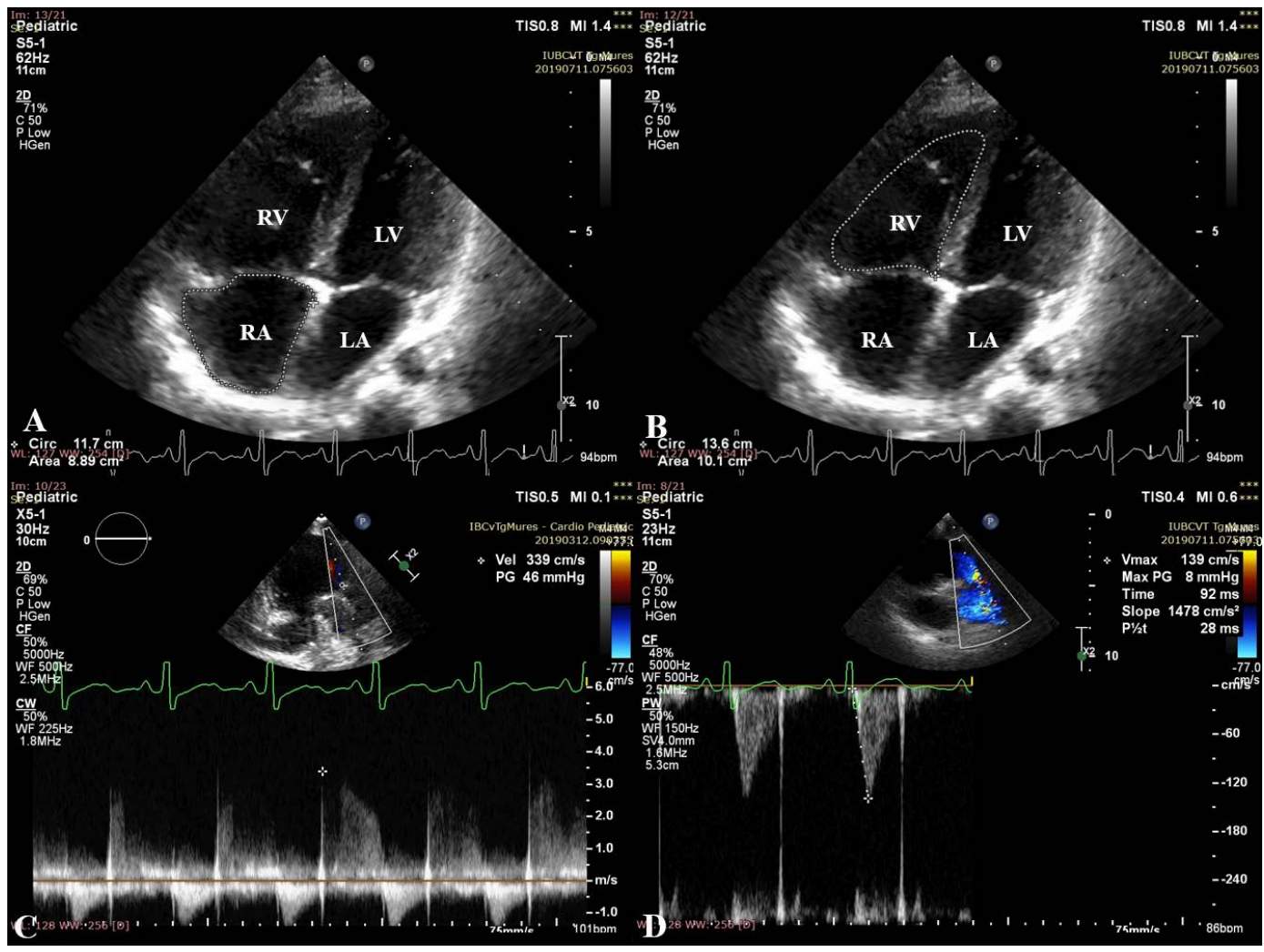

FIGURE 3. Echocardiographic views 6 months after initiation of vasodilator therapy demonstrating regression of the right cavities and improvement of echocardiographic parameters; $\mathbf{A}$ - right atrial area of $8.89 \mathrm{~cm}^{2}$; B - right ventricular area of $10.1 \mathrm{~cm}^{2} ; \mathbf{C}$ - mean pulmonary arterial pressure of $46 \mathrm{mmHg}$; D - pulmonary artery acceleration time of 92 ms. LA - left atrium; LV - left ventricle; RA - right atrium; RV - right ventricle 


\section{DISCUSSIONS}

Late-onset PAH after successful neonatal ASO for simple TGA is a rare, but potentially life-threatening complication. The association between PAH and TGA was first recognized as a complication after ASO, multiple mechanisms being related to the development of $\mathrm{PAH}$ in these patients. ${ }^{5}$

The pathophysiological mechanisms that lead to PAH in children with TGA after timely ASO in the absence of hemodynamically relevant residual lesions are not fully understood. PAH onset may be early or late after ASO. ${ }^{5}$ The proposed mechanisms may include genetic susceptibility and abnormal prenatal and postnatal pulmonary hemodynamics. ${ }^{5}$ Several genes, such as ACKR3 (or CXCR7) and NF1, have been associated both with TGA and with the development of PAH on the strength of endothelial dysfunction and pulmonary vascular remodeling., ${ }^{5,10,11}$ Prenatal hemodynamic alterations, including restriction of the foramen ovale or ductus arteriosus, are associated with hypoxia of the prenatal pulmonary circulation and increased bronchial circulation., ${ }^{5,12-14}$ Altered prenatal pulmonary hemodynamics is associated with PPHN, a condition of disturbed adaptation of the pulmonary vasculature to postnatal life. ${ }^{1,5}$ PPHN occurs in $1-4 \%$ of neonates with TGA with IVS, and it is associated with higher rates of mortality and postoperative complications. ${ }^{15}$ It has been suggested that PPHN is associated with the development of PAH later in life. ${ }^{5}$ Several pathophysiological mechanisms have been proposed regarding the development of $\mathrm{PAH}$ after ASO, including postnatal hyperoxic blood perfusing the pulmonary vasculature in uncorrected TGA, the dispersion of microthrombi during atrial balloon septostomy, and the vulnerability of the pulmonary vascular bed during the immediate postoperative period. ${ }^{5}$ Prenatal hypoxia and postnatal hyperoxia within the pulmonary vasculature in TGA may injure or trigger pulmonary vasculature remodeling, thus PAH may develop early or later after ASO. ${ }^{5}$

All patients who have undergone TGA repair should have long-term follow-up care by a cardiologist with expertise in congenital heart disease. Echocardiography is the recommended screening technique for $\mathrm{PAH}$, while right heart catheterization is the gold standard method for diagnosing $\mathrm{PAH}$, and also it can be used to guide treatment strategies. Other imaging investigations, such as computed tomography, are extremely useful both in evaluating possible residual lesions and in evaluating the pulmonary parenchyma. Despite the intense use of PAHtargeted therapies, the prognosis is poor and comparable with that of children with idiopathic PAH. ${ }^{5}$
The incidence of PAH after ASO has not been well described as there have been several cases reported in the literature. Based on the literature review, Zijlstra et al. reported an estimated incidence of $0.6-1 \%$ for $\mathrm{PAH}$ after neonatal ASO for TGA. ${ }^{5}$ In their retrospective study, they identified 25 children with TGA that developed severe, progressive PAH after timely and successful ASO and in the absence of hemodynamically relevant residual lesions. ${ }^{5}$ Of these patients, 8 children presented with lateonset PAH, years after ASO, and 17 children with earlyonset PAH, at weeks or months after ASO. ${ }^{5}$ Three children $(13 \%)$ had associated PPHN. Their study could not identify risk factors for the development of PAH after ASO for TGA, but the role of altered prenatal pulmonary hemodynamics in TGA has been speculated. ${ }^{5}$

Our case describes a female pediatric patient who underwent ASO for simple TGA with IVS in the neonatal period, without remaining defects, and who developed PAH at the age of 5 years. This case belongs to the fourth subgroup of $\mathrm{PAH}$ associated with congenital heart disease, according to the classification of $\mathrm{PAH} .{ }^{1}$ We reported a favorable treatment response, defined as the improvement of the 6-minute walk test, WHO FC, and echocardiographic parameters at 6 months. Our patient is the first reported Romanian patient with late-onset PAH after neonatal ASO for simple TGA.

To the best of our knowledge, a small number of patients with late-onset PAH after ASO were successfully treated with vasodilators. Similar to our patient, Torres et al. and Cordinia et al. reported favorable response up to a year after the initiation of a combination of bosentan and sildenafil in a 9-year-old and 16-year-old male respectively, both with severe late-onset PAH. ${ }^{16,17}$ Monotherapy with sildenafil proved to be beneficial in a 7-year-old boy with PAH after ASO. ${ }^{18}$ Smedema et al. described a young male patient who was diagnosed with severe PAH 14 years after neonatal ASO.19

\section{CONCLUSIONS}

Severe PAH is a rarely reported complication after ASO, and the development of PAH after ASO is still not well understood. Patients after TGA repair should be followed regularly with clinical and echocardiographic assessment in order to identify the development of PAH, because early diagnosis and aggressive combination therapy for $\mathrm{PAH}$ could help to improve survival in these patients.

\section{CONFLICT OF INTEREST}

Nothing to declare. 


\section{REFERENCES}

1. Simonneau G, Montani D, Celermajer DS, et al. Haemodynamic definitions and updated clinical classification of pulmonary hypertension. Eur Respir J. 2019;53:1801913. doi: 10.1183/13993003.01913-2018.

2. Reller MD, Strickland MJ, Riehle-Colarusso T, Mahle WT, Correa A. Prevalence of congenital heart defects in metropolitan Atlanta, 1998-2005. J Pediatr. 2008;153:807813. doi: 10.1016/j.jpeds.2008.05.059.

3. Jatene $\mathrm{AD}$, Fontes VF, Paulista $\mathrm{PP}$, et al. Anatomic correction of transposition of the great vessels. J Thorac Cardiovasc Surg. 1976;72:364.

4. Khairy P, Clair M, Fernandes SM, et al. Cardiovascular outcomes after the arterial switch operation for D-transposition of the great arteries. Circulation. 2013;127:331-339. doi: 10.1161/ CIRCULATIONAHA.112.135046.

5. Zijlstra WM, Elmasry O, Pepplinkhuizen S, et al. Pulmonary arterial hypertension in children after neonatal arterial switch operation. Heart. 2017;103:1244-1249. doi: 10.1136/ heartjnl-2016-310624.

6. Sarris GE, Balmer C, Bonou P, et al. Clinical guidelines for the management of patients with transposition of the great arteries with intact ventricular septum. Cardiol Young. 2017;27:530-569. doi: 10.1017/S1047951117000014.

7. Losay J, Touchot A, Serraf A, et al. Late outcome after arterial switch operation for transposition of the great arteries. Circulation. 2001;104:I121-I126. doi: 10.1161/hc37t1.094716.

8. Angeli E, Raisky O, Bonnet D, Sidi D, Vouhé PR. Late reoperations after neonatal arterial switch operation for transposition of the great arteries. Eur J Cardiothorac Surg. 2008;34:32-36. doi: 10.1016/j.ejcts.2008.04.007.

9. Watanabe T, Adachi O, Suzuki Y, et al. Lung Transplant for Pulmonary Arterial Hypertension After Arterial Switch Operation. Ann Thorac Surg. 2015;100:e133-4. doi: 10.1016/j. athoracsur.2015.08.046.
10. Costello CM, McCullagh B, Howell K, et al. A role for the CXCL12 receptor, CXCR7, in the pathogenesis of human pulmonary vascular disease. Eur Respir J. 2012;39(6):14151424. doi: 10.1183/09031936.00044911.

11. Montani D, Coulet F, Girerd B, et al. Pulmonary hypertension in patients with neurofibromatosis type I. Medicine (Baltimore). 2011;90:201-211. doi: 10.1097/MD.0b013e31821be2b7.

12. Newfeld EA, Paul MH, Muster AJ, et al. Pulmonary vascular disease in transposition of the great vessels and intact ventricular septum. Circulation. 1979;59:525-530. doi: 10.1161/01.cir.59.3.525.

13. Wernovsky G, Bridges ND, Mandell VS, et al. Enlarged bronchial arteries after early repair of transposition of the great arteries. J Am Coll Cardiol. 1993;21:465-470. doi: 10.1016/0735-1097(93)90690-3.

14. Rudolph AM. Aortopulmonary transposition in the fetus: speculation on pathophysiology and therapy. Pediatr Res. 2007;61:375-380. doi: 10.1203/pdr.ob013e318030d5b9.c.

15. Sallaam S, Natarajan G, Aggarwal S. Persistent Pulmonary Hypertension of the Newborn with D-transposition of the Great Arteries: Management and Prognosis. Congenit Heart Dis. 2016;11:239-244. doi: 10.1111/chd.12304.

16. Torres M, Coserria JF, Gavilan JL. Late development of pulmonary arterial hypertension after arterial switch for transposition of the great arteries. Scientific letters/Rev Esp Cardiol. 2012;65:1054-1065. doi: 10.1016/j.recesp.2012.03.021.

17. Cordina R, Celermajer D. Late-onset pulmonary arterial hypertension after a successful atrial or arterial switch procedure for transposition of the great arteries. Pediatr Cardio. 2010;31:238-241. doi: 10.1007/s00246-009-9597-9.

18. Carroll WD, Dhillon R. Sildenafil as a treatment for pulmonary hypertension. Arch Dis Child. 2003;88:827-828. doi: 10.1136/ adc.88.9.827.

19. Smedema J-P, Pistorius L, Brink J. Late-onset pulmonary arterial hypertension after successful early arterial switch surgery for simple transposition of the great arteries. SA Heart. 2016;13:28-32. doi: 10.24170/13-1-1689. 